LETTER • OPEN ACCESS

\section{Origin of the material dependence of temperature coefficient of redox potential in conjugated polymers}

To cite this article: Hiroki Iwaizumi et al 2021 Appl. Phys. Express 14037001

View the article online for updates and enhancements.
You may also like

- Pressure driven long wavelength MHD
$\frac{\text { instabilities in an axisymmetric toroidal }}{\text { resistive plasma }}$
J P Graves, M Coste-Sarguet and C
Wahlberg
- Correlation between coherent charge
$\frac{\text { transport and crystallinity in doped - }}{\text { coniugated polymers }}$
Kan Ueji, Masahiro Ohno, Jun Takeya et
al.
- Weak measurement-based state
$\frac{\text { estimation of Gaussian states of one- }}{\text { variable quantum systems }}$
Debmalya Das and Arvind




\title{
Origin of the material dependence of temperature coefficient of redox potential in conjugated polymers
}

\author{
Hiroki Iwaizumi ${ }^{1}$, Dai Inoue ${ }^{1}$, Takeshi Yasuda ${ }^{2}$, and Yutaka Moritomo ${ }^{1,3,4^{*}}$ \\ ${ }^{1}$ Graduate School of Pure and Applied Science, University of Tsukuba, Tennodai 1-1-1, Tsukuba, Ibaraki 305-7571, Japan \\ ${ }^{2}$ Research Center for Functional Materials, National Institute for Materials Science (NIMS), Tsukuba 305-0047, Japan \\ ${ }^{3}$ Faculty of Pure and Applied Science, University of Tsukuba, Tennodai 1-1-1, Tsukuba, Ibaraki 305-7571, Japan \\ ${ }^{4}$ Tsukuba Research Center for Energy Materials Science (TREMS), University of Tsukuba, Tsukuba, Ibaraki 305-8571, Japan \\ *E-mail: moritomo.yutaka.gf@u.tsukuba.ac.jp \\ Received December 25, 2020; revised January 12, 2021; accepted January 28, 2021; published online February 23, 2021
}

The thermal coefficient $\alpha(=d V / d T)$ of the redox potential $(V)$ is an important physical parameter for thermal energy harvesting. To clarify the microscopic origin of $\alpha$ in conjugated polymers, we compared $\alpha$ and the physical quantity obtained by a quantum chemistry calculation in typical polymers with small monomer molecular weight. We observed a strong correlation between $\alpha$ and the number ( $N_{\text {active }}$ ) of the active atom, which is defined as the atom whose variation of the Mulliken charge $(\Delta q)$ at the oxidation process is beyond a threshold $\left(\Delta q_{\mathrm{th}}\right)$. We interpreted the material dependence of $\alpha$ in terms of $N_{\text {active, }}$, because active atoms have a significant effect on the configuration entropy of the surrounding solvent molecules. (C) 2021 The Author(s). Published on behalf of The Japan Society of Applied Physics by IOP Publishing Ltd

Supplementary material for this article is available online

$t$ is crucial to develop thermal energy-harvesting devices that produce electrical energy efficiently and inexpensively from environmental waste heat, to realize a "smart" society. Recently, several researchers reported that a battery can be charged by environmental heat by using the difference $(\Delta \alpha)$ in the thermal coefficient $\alpha(=d V / d T)$ of the redox potential $(V)$ between the cathode and anode materials. ${ }^{1-8)}$ The battery can produce electrical energy in thermal cycles between the low $\left(T_{\mathrm{L}}\right)$ and high $\left(T_{\mathrm{H}}\right)$ temperatures and is called a tertiary battery. In the warming process, the cell voltage $\left(V_{\text {cell }}\right)$ increases when heated from $0 \mathrm{~V}$ to $\Delta \alpha \Delta T$ $\left(\Delta T=T_{\mathrm{H}}-T_{\mathrm{L}}\right)$. In other words, the battery is charged by the environmental heat. The accumulated electrical energy can be extracted by the discharging process at $T_{\mathrm{H}}$. Similarly, $V_{\text {cell }}$ decreases with cooling from $0 \mathrm{~V}$ to $-\Delta \alpha \Delta T$. Shibata et al. ${ }^{4)}$ fabricated a tertiary battery consisting of two kinds of cobalt Prussian blue analogs (Co-PBAs) with different $\alpha$, i.e. $\mathrm{Na}_{x} \mathrm{Co}\left[\mathrm{Fe}(\mathrm{CN})_{6}\right]_{0.71}$ (NCF71) and $\mathrm{Na}_{x} \mathrm{Co}\left[\mathrm{Fe}(\mathrm{CN})_{6}\right]_{0.90}$ (NCF90), and aqueous electrolyte. The NCF71/NCF90 tertiary battery produces electrical energy with high thermal efficiency $(\eta=1 \%)$ between $T_{\mathrm{L}}(=295 \mathrm{~K})$ and $T_{\mathrm{H}}(=323 \mathrm{~K})$. They further increased the $V_{\text {cell }}$ with the use of a phase transition of Co-PBA. ${ }^{8)}$ The $V_{\text {cell }}$ of the $\mathrm{Na}_{x} \mathrm{Co}\left[\mathrm{Fe}(\mathrm{CN})_{6}\right]_{0.82}$ (NCF82)/NCF90 tertiary battery steeply increased from 0 to $120 \mathrm{mV}$ at $\sim 320 \mathrm{~K}$.

From a thermodynamic point of view, $\alpha$ is expressed as $\frac{\Delta S}{e}$, where $e(\geqslant 0)$ and $\Delta S$ are the elementary charge and difference in entropies $(S)$ between the reduced and oxidized states, respectively. Importantly, the redox process in a battery system influences not only the solid electrode, but the electrolyte. In other words, $\Delta S$ consists of the solid ( $\left.\Delta S_{\text {solid }}\right)$ and electrolyte ( $\Delta S_{\text {electrolyte }}$ ) components. ${ }^{9)}$ Generally, $\Delta S_{\text {electrolyte }}$ is ascribed to the variation in the configuration entropy of the solvent molecules.

Among the battery materials, conjugated polymers have several advantages for thermal energy harvesting, such as light weight, flexibility and environmental friendliness. ${ }^{10-13)}$ Many conjugated polymers, e.g. polyacetylene, ${ }^{14)}$ poly(p-phenylene) (PPP) ${ }^{15,16)}$ and polythiophene, ${ }^{17)}$ show the reversible redox process among the reduced, neutral and oxidized states. The characteristic of conjugated polymers is that $\alpha$ strongly depends on the main chain structure; ${ }^{18)} \alpha=1.08 \mathrm{mVK}^{-1}$ for poly(3-hexylthiophene2,5-diyl) (P3HT), $0.38 \mathrm{mVK}^{-1}$ for poly(9,9-dioctylfluorene-co-bithiophene) (F8T2) and $0.19 \mathrm{mVK}^{-1}$ for poly [9,9-dioctylfluorene-co-N-(4-butylphenyl) diphenylamine] (TFB). In our previous work, ${ }^{18}$ the material dependence is interpreted in terms of the vibrational entropy of the benzene $\left(\mathrm{C}_{6} \mathrm{H}_{5}\right)$ and thiophene $\left(\mathrm{C}_{4} \mathrm{H}_{4} \mathrm{~S}\right)$ units. In this model, the main chains of the polymers were approximated to be composed of the $\mathrm{C}_{6} \mathrm{H}_{5}$ and $\mathrm{C}_{4} \mathrm{H}_{4} \mathrm{~S}$ units. The model, however, may be too simple to describe the actual materials. In addition, the model is not applicable to other polymers. Therefore, we need to build a more realistic model to comprehend the material dependence of $\alpha$.

In this work, we investigated four typical conjugated polymers with small monomer molecular weight, i.e. (a) Poly(4-butylphenyl-diphenylamine) (PTAA), (b) poly(2,5dioctyl-1,4-phenylene) (PPP), (c) poly[2-methoxy-5-(2-ethylhexyloxy)-1,4-phenylenevinylene] (MEH-PPV) and (d) P3HT (see Fig. 1). We compared $\alpha$ and the physical quantity obtained by a quantum chemistry calculation on the monomer unit including the side chains. We observed a strong correlation between $\alpha$ and the number $\left(N_{\text {active }}\right)$ of the active atom, which is defined as the atom whose variation of the Mulliken charge $(\Delta q)$ at the oxidation process is beyond a threshold $\left(\Delta q_{\mathrm{th}}\right)$. We interpreted the material dependence of $\alpha$ in terms of $N_{\text {active, }}$ because the active atoms have a significant effect on the configuration entropy of the neighboring solvent molecules.

We prepared PTAA according to methods in the literature. ${ }^{19)}$ We purchased MEH-PPV from Sigma-Aldrich and PPP from American Dye source, which were all used as received. Polymer films were prepared by casting the polymer solution on a glass substrate coated with indium tin oxide (ITO) electrode (GEOMATEC Co., Ltd.). The 


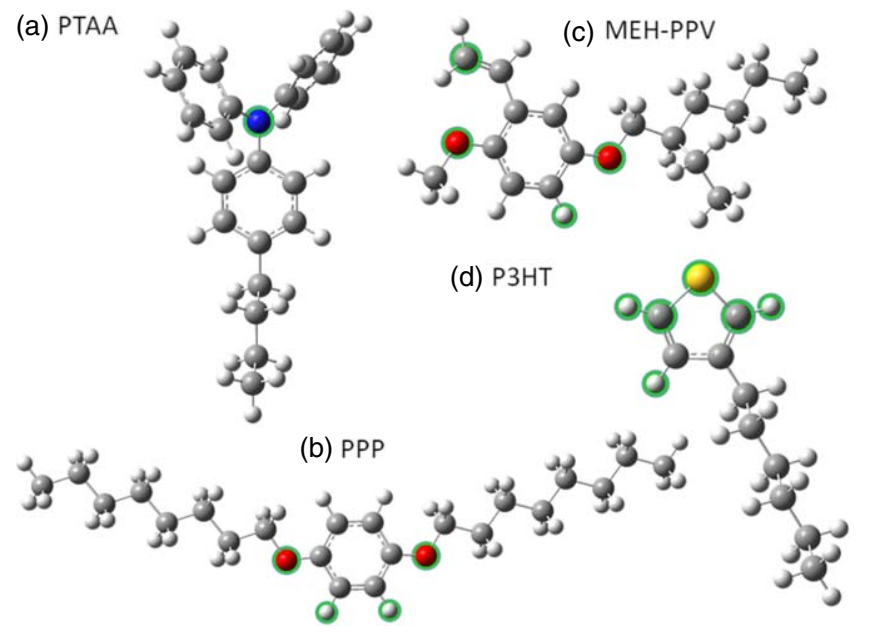

Fig. 1. (Color online) Monomer structure of (a) PTAA, (b) MEH-PPV, (c) PPP and (d) P3HT. Structures were optimized at the B3LYP/6-311G $(p, d)$ level. Atom surrounded by a green circle represents the active atoms (see text).

thickness and sheet resistance of the ITO film were $150 \mathrm{~nm}$ and $\leqslant 10 \Omega \mathrm{sq}^{-1}$, respectively. The thickness of the glass substrate was $0.7 \mathrm{~mm}$. For PTAA and MEH-PPV, $2.5 \mathrm{mg}$ of polymer was dissolved in $0.5 \mathrm{ml}$ of chloroform. The thickness and weight of the films were typically $6 \mu \mathrm{m}$ and $0.04 \mathrm{mg}$, respectively. For PPP, $25 \mathrm{mg}$ of PPP was dissolved in $0.5 \mathrm{ml}$ of chloroform to stabilize the redox potential. The polymer weight was evaluated from the weight of the polymer solution cast on the substrate. The areas of the electrodes are about $0.3 \mathrm{~cm}^{2}$. The electrochemical measurements were performed using a beaker-type cell with a potentiostat (HokutoDENKO; HJ1001SD8) within an Arfilled glove box. The cathode, anode and electrolyte were the cast film, Li metal, and ethylene carbonate (EC)/diethyl carbonate (DEC) containing $1 \mathrm{~mol} / 1 \mathrm{LiClO}_{4}$, respectively. The charge and discharge rate were $0.3 \mathrm{C}$ for PTAA and PPP and $0.9 \mathrm{C}$ for $\mathrm{MEH}-\mathrm{PPV}$. The charge/discharge rate is defined by the reciprocal of the time $(h)$ required for full charge/discharge. The corresponding currents were 0.44, 0.56 and $6.20 \mu \mathrm{A}$ for PTAA, PPP and MEH-PPV, respectively. The upper and lower cut-off voltages were set to be 3.8-4.0 $\mathrm{V}$ and $1.0 \mathrm{~V}$ vs $\mathrm{Li} / \mathrm{Li}^{+}$, respectively.

We determined $\alpha_{\text {cell }}$ of the beaker-type cell, whose cathode, anode and electrolyte were the cast film, Li metal, and EC/DEC containing $1 \mathrm{~mol} / \mathrm{LiClO}_{4}$, respectively. $\alpha$ of the conjugated polymer is expressed as $\alpha_{\text {cell }}-\alpha_{\mathrm{Li}}$, where $\alpha_{\text {cell }}$ and $\alpha_{\mathrm{Li}}$ are the coefficient of the cell and $\mathrm{Li}$ metal, respectively. $\alpha_{\mathrm{Li}}$ in EC/DEC containing $1 \mathrm{~mol} / 1 \mathrm{LiClO}_{4}$ is $0.76 \mathrm{mVK}^{-1}$.9) To avoid the potential fluctuation that is prominent in the as-grown film, the polymers are partially oxidized (arrows in Fig. 2) in the beaker-type cell. $V_{\text {cell }}$ was carefully measured against the cell temperature $(T)$, which was monitored with a $\mathrm{Pt}$ resistance thermometer in the electrolyte. $T$ was slowly increased/decreased at a rate of $\pm 0.3 \mathrm{Kmin}^{-1}$.

Figure 2 shows the charge and discharge curves of the cast films: (a) PTAA, (b) PPP and (c) MEH-PPV. In the PTAA film [(a)], the discharge curve shows a single plateau at $3.7 \mathrm{~V}$, which is assigned to the removal of an electron from the highest occupied molecular orbital (HOMO). Similar single
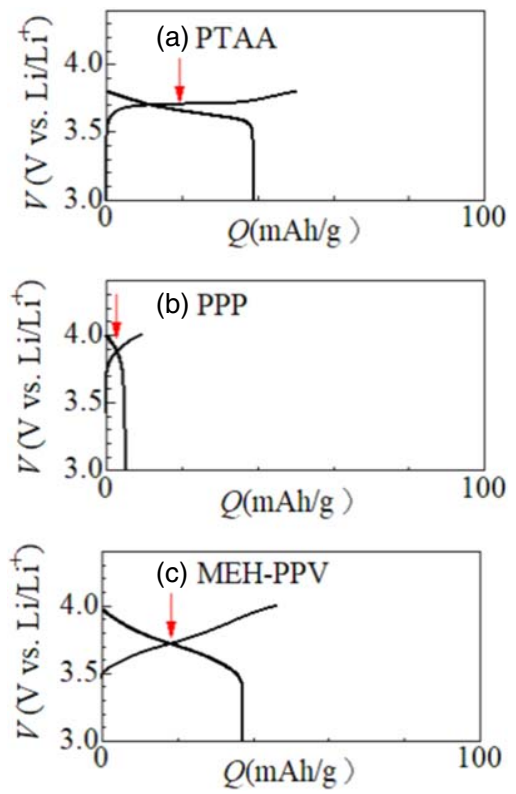

Fig. 2. (Color online) Charge and discharge curves of the cast films against the charge (Q): (a) PTAA, (b) PPP and (c) MEH-PPV. Charge and discharge rate were $0.3 \mathrm{C}$ (PTAA), $0.3 \mathrm{C}(\mathrm{PPP})$ and $0.9 \mathrm{C}(\mathrm{MEH}-\mathrm{PPV})$. Arrows indicate the positions where $\alpha$ was determined.

plateaus are observed in the PPP [(b)] and MEH-PPV [(c)] cast films and are assigned to the removal of an electron from the HOMOs. The discharge capacities $\left(Q_{\text {exp }}\right)$ were evaluated and are listed in Table I together with the ideal value $\left(Q_{\text {ideal }}\right)$. Except for P3TH, $Q_{\exp }$ is much smaller than $Q_{\text {ideal }}$, indicating that these polymers can be only partially oxidized.

Figure 3 shows $V_{\text {cell }}$ of the Li/polymer cell against $T$ : (a) PTAA, (b) PPP and (c) MEH-PPV. Red and blue marks represent the data observed in the heating and cooling runs, respectively. No thermal hysteresis was observed in $V_{\text {cell }}$, which indicates that the temperature gradient as well as the sample deterioration are excluded. $\alpha_{\text {cell }}$ of the Li/polymer cell was evaluated by least-squares fitting (straight lines in Fig. 3). $\alpha$ of the conjugated polymer was obtained using the relation, $\alpha=\alpha_{\text {cell }}+0.76 \mathrm{mVK}^{-1}$, and listed in Table I.

First, let us consider the vibrational component $\left(\alpha_{\mathrm{vib}}\right)$ of $\alpha$, which is expressed as $\frac{S_{\text {vib }}^{\text {neu }}-S_{\text {vib }}^{\text {oxi }}}{e}$, where $S_{\text {vib }}^{\text {neu }}$ and $S_{\text {vib }}^{\text {oxi }}$ are the vibrational entropies in the neutral and oxidized states, respectively. In the oxidation process, an electron with bonding character is removed from the HOMO. The removal of an electron weakens the force constant between the neighboring atoms and lowers the frequencies of the vibrational modes. ${ }^{20-23)}$ The softening of the modes increases the number of thermally-excited phonons, and hence, increases $S_{\text {vib }}^{\text {oxi }}$ To evaluate $S_{\text {vib }}^{\text {neu }}\left(S_{\text {vib }}^{\text {oxi }}\right)$, quantum chemistry calculations were performed on the monomer unit with including side

Table I. Observed $\left(Q_{\text {exp }}\right)$ and ideal $\left(Q_{\text {ideal }}\right)$ capacities of conjugated polymers together with the temperature coefficient $(\alpha)$ of redox potential. $Q_{\text {ideal }}$ was evaluated assuming that one electron can be accommodated/ removed per monomer unit. Data of P3HT are cited from Ref. 18.

\begin{tabular}{lccc}
\hline Polymer & $Q_{\exp }\left(\mathrm{mAhg}^{-1}\right)$ & $Q_{\text {ideal }}\left(\mathrm{mAhg}^{-1}\right)$ & $\alpha\left(\mathrm{mVK}^{-1}\right)$ \\
\hline PTAA & 39 & 89.5 & 0.02 \\
PPP & 5 & 80.6 & 0.41 \\
MEH-PPV & 37 & 102.9 & 0.46 \\
P3HT & 90 & 162.5 & 1.08 \\
\hline
\end{tabular}

(C) 2021 The Author(s). Published on behalf of 

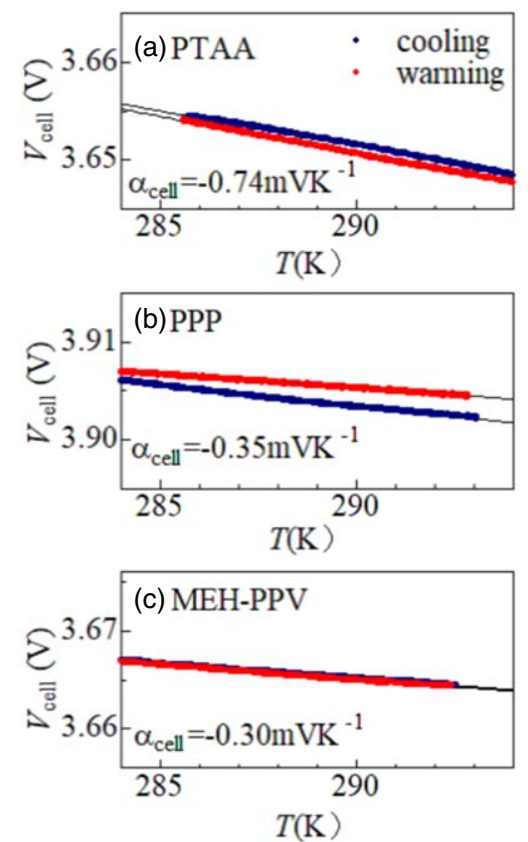

Fig. 3. (Color online) Cell voltage $\left(V_{\text {cell }}\right)$ of the Li/polymer cell against the cell temperature (T): (a) PTAA, (b) PPP and (c) MEH-PPV. Blue and red marks represent the data obtained in the cooling and warming runs, respectively. Straight lines are the results of the least-squares fitting. In (b) PPP and (c) MEH-PP, a decrease in $V_{\text {cell }}$ was observed in proportion to time $(t)$ and was corrected with a $t$-linear function.

chains in the neutral (oxidized) state. The calculations were performed with Gaussian16 at the B3LYP/6-311G $(d, p)$ level. $^{24)}$ After the structural optimization (Fig. 1), the frequencies of the vibrational modes were evaluated (Table S1; supplementary data, available online at stacks. iop.org/APEX/14/037001/mmedia). The number of thermally-excited phonons is proportional to $e^{-\frac{\hbar \omega_{i}}{k_{\mathrm{B}} T}}$, where $\hbar \omega_{i}$ and $k_{\mathrm{B}}$ are the vibrational energy of the $i$ th mode and Boltzmann constant, respectively. Hereafter, we use the vibrational energy $x_{i}\left(=\frac{\hbar \omega_{i}}{k_{\mathrm{B}} T}\right)$ as the unit of thermal energy. After a simple calculation, $S_{\text {vib }}$ is expressed as $k_{\mathrm{B}} \sum_{i=1}^{N}\left[\frac{x_{i} e^{-x_{i}}}{1-e^{-x_{i}}}-\ln \left(1-e^{-x_{i}}\right)\right]$, where $N$ is the number of vibrational modes. We note that $\alpha_{\text {vib }}$ does not include the low-energy lattice modes, e.g. the translational or rotational movement of the entire monomer and movement of the side chain relative to the main chain. In addition, $\alpha_{\text {vib }}$ does not include the configuration entropy of the side chains. The reduction process, however, is considered to have a negligible effect on the low-energy lattice mode as well as the configuration entropy of the side chains.

Table II. Vibrational entropies in the neutral $\left(S_{\text {vib }}^{\text {neu }}\right)$ and oxidized $\left(S_{\text {vib }}^{\text {oxi }}\right)$ states of monomers at $298.15 \mathrm{~K}$. Vibrational component $\left(\alpha_{\mathrm{vib}}\right)$ of $\alpha$ is expressed as $\frac{S_{\mathrm{vib}}^{\mathrm{neu}}-S_{\mathrm{vib}}^{\text {oxi }}}{e} . \alpha$ is the experimental value. $\alpha$ of P3HT is cited from Ref. 18.

\begin{tabular}{lcccc}
\hline Monomer & $\begin{array}{c}S_{\text {vib }}^{\text {neu }} \\
\left(\mathrm{meVK}^{-1}\right)\end{array}$ & $\begin{array}{c}S_{\text {vib }}^{\text {oxi }} \\
\left(\mathrm{meVK}^{-1}\right)\end{array}$ & $\begin{array}{c}\alpha_{\text {vib }} \\
\left(\mathrm{mVK}^{-1}\right) .\end{array}$ & $\alpha\left(\mathrm{mVK}^{-1}\right)$ \\
\hline PTAA & 3.402 & 3.289 & 0.113 & 0.02 \\
PPP & 5.273 & 5.298 & -0.025 & 0.41 \\
MEH-PPV & 3.512 & 3.574 & -0.062 & 0.46 \\
P3HT & 1.803 & 1.839 & -0.036 & 1.08 \\
\hline
\end{tabular}

In Table II, we listed $S_{\text {vib }}^{\text {neu }}, S_{\text {vib }}^{\text {oxi }}$ and $\alpha_{\text {vib }}$, together with the experimental value $(\alpha)$. We found that $\alpha_{\mathrm{vib}}$ is an order of magnitude smaller than $\alpha$, suggesting that $\alpha_{\text {vib }}$ at least is not the main origin of the material dependence of $\alpha$. In addition, the order of $S_{\text {vib }}$ is opposite to the order of $\alpha$, indicating that $S_{\text {vib }}$ cannot qualitatively explain the material dependence of $\alpha$. Therefore, we need to search the origin for the material dependence in another physical parameter. In our previous work, ${ }^{18)}$ the material dependence was explained by the fact that $\alpha_{\text {vib }}$ of thiophane $\left(\mathrm{C}_{4} \mathrm{H}_{4} \mathrm{~S}\right)$ is larger than that of benzene $\left(\mathrm{C}_{6} \mathrm{H}_{5}\right)$. In this model, the main chains of the polymers were approximated to be composed of the $\mathrm{C}_{6} \mathrm{H}_{5}$ and $\mathrm{C}_{4} \mathrm{H}_{4} \mathrm{~S}$ units. In the actual conjugated polymers, however, an electron escapes not only from a single $\mathrm{C}_{6} \mathrm{H}_{5}$ or $\mathrm{C}_{4} \mathrm{H}_{4} \mathrm{~S}$ unit, but from many atoms in a strongly material-dependent manner (Table S2). In this sense, this model was too simple to describe the actual materials.

Here, we note that $\Delta S$ is dominated by $\Delta S_{\text {electrolyte }}$ in a solute system, e.g. $\left[\mathrm{Fe}(\mathrm{CN})_{6}\right]^{4-} /\left[\mathrm{Fe}(\mathrm{CN})_{6}\right]^{3-}$ in water. ${ }^{25)}$ In this system, $\Delta S_{\text {electrolyte }}$ is evaluated to be $-1.28 \mathrm{mVK}^{-1}$ since $\Delta S$ originated in $\left[\mathrm{Fe}(\mathrm{CN})_{6}\right]^{4+} /\left[\mathrm{Fe}(\mathrm{CN})_{6}\right]^{3+}$ is $-0.22 \mathrm{mVK}^{-1} \cdot{ }^{26,27)}$ In general, $\Delta S_{\text {electrolyte }}$ is ascribed to the variation in the configuration entropy of solvent molecules around the redox couple. By means of quantum mechanical and molecular dynamics calculations, Prampolini et al. ${ }^{28)}$ investigated the water structure around $\left[\mathrm{Fe}(\mathrm{CN})_{6}\right]^{4-} /\left[\mathrm{Fe}(\mathrm{CN})_{6}\right]^{3-}$. They found that water molecules get closer to $\left[\mathrm{Fe}(\mathrm{CN})_{6}\right]^{4-}$ than to $\left[\mathrm{Fe}(\mathrm{CN})_{6}\right]^{3-}$, reflecting the higher charge of $\left[\mathrm{Fe}(\mathrm{CN})_{6}\right]^{4-}$. In order to evaluate the effect of the configuration entropy of the EC and DEC molecules, we experimentally investigated $\alpha$ of $\mathrm{Fe}^{2+} / \mathrm{Fe}^{3+}$ in EC/DEC (Fig. S1; supplementary data). We found that the corresponding $\alpha\left(=2.21 \mathrm{mVK}^{-1}\right)$ is large, indicating that the configuration entropy of the EC and DEC molecules significantly changes around $\mathrm{Fe}^{2+} / \mathrm{Fe}^{3+}$ in the redox process. A similar effect is expected even in the polymer system, because most of the polymers are in contact with the electrolyte. In other words, the $\Delta S_{\text {electrolyte }}$ component is expected if the variation $(\Delta q)$ of the Mulliken charges for the constituent atom at the oxidation process is large. Table S2 (supplementary data) shows the Mulliken charges in PTAA, MEH-PPV, PPP and P3HT in neutral $\left(q^{\text {neu }}\right)$ and oxidized $\left(q^{\text {oxi }}\right)$ states. The serial numbers of the atoms are shown in Fig. S2 (supplementary data).

Figure 4(a) shows $\Delta q\left(=q^{\text {oxi }}-q^{\text {neu }}\right)$ in the oxidation process for $\mathrm{S}, \mathrm{N}, \mathrm{O}$ (filled symbols) and $\mathrm{C}$ (open symbols).
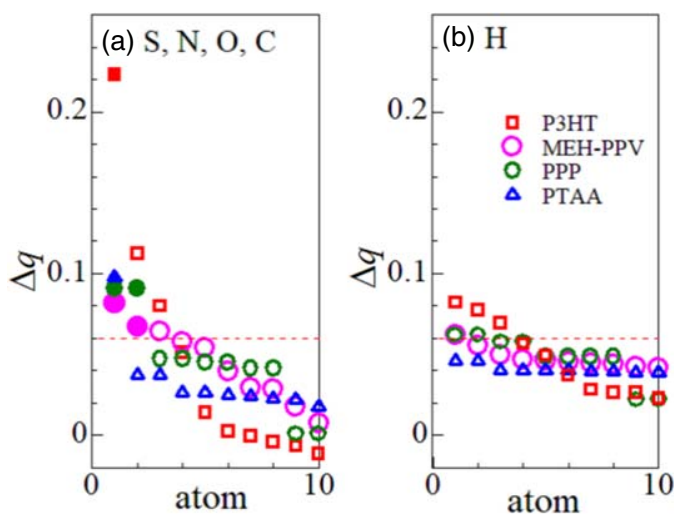

Fig. 4. (Color online) Variation $(\Delta q)$ of the Mulliken charge in the oxidation process; (a) S, N, O and C and (b) H. $\Delta q$ s are shown in order of quantity. Open (closed) symbols in (a) represent $\mathrm{C}(\mathrm{S}, \mathrm{N}, \mathrm{O})$. Broken red line means a tentative threshold $\left(\Delta q_{\mathrm{th}}\right)$.

(C) 2021 The Author(s). Published on behalf of 037001-3 The Japan Society of Applied Physics by IOP Publishing Ltd 
Table III. Number $\left(N_{\text {active }}\right)$ of the active atoms in monomers with different threshold $\left(\Delta q_{\mathrm{th}}\right)$.

\begin{tabular}{lcccc}
\hline$\Delta q_{\text {th }}$ & P3HT & MEH-PPV & PPP & PTAA \\
\hline 0.08 & 3 & 1 & 2 & 1 \\
0.07 & 5 & 1 & 2 & 1 \\
0.06 & 6 & 4 & 4 & 1 \\
0.05 & 8 & 8 & 6 & 1 \\
\hline
\end{tabular}

$\Delta q$ s are shown in order of quantity. In PTAA (triangles), $\Delta q$ is very small; $\Delta q$ s from the largest are $0.10,0.04$, etc. We found that $\Delta q$ is much enhanced in P3HT (squares); $\Delta q$ s from the largest are $0.22,0.12,0.08,0.05$, etc. A similar trend is observed in $\Delta q$ for $\mathrm{H}$ [Fig. 4(b)]. The atom with large $\Delta q$ significantly influences the configuration entropy of the surrounding solvent molecules. We will call such an atom an "active atom". Here, we introduce a threshold $\left(\Delta q_{\mathrm{th}}\right)$ to identify an atom with large $\Delta q\left(\geqslant \Delta q_{\mathrm{th}}\right)$. The broken red line in Fig. 4 is an example of $\Delta q_{\mathrm{th}}(=0.06)$. In this case, the number $\left(N_{\text {active }}\right)$ of the active atoms are one for (a) PTAA, four for (b) PPP and (c) MEH-PPV and six for (d) P3HT. The active atoms are indicated by green circles in Fig. 1. The order of $N_{\text {active }}$ is the same order as $\alpha$, suggesting that the material dependence can be ascribed to $N_{\text {active. }}$. We recognized that $\Delta q_{\text {th }}$ is only a guide and has no absolute physical meaning. We carefully investigated $\Delta q_{\mathrm{th}}$-dependence of $N_{\text {active }}$ (Table III). We confirmed that the order of $N_{\text {active }}$ is insensitive to $\Delta q_{\mathrm{th}}$.

In conclusion, we compared $\alpha$ and the physical quantity obtained by a quantum chemistry calculation on the monomer unit including the side chains. We observed a strong correlation between $\alpha$ and $N_{\text {active, suggesting that the material dependence of }}$ $\alpha$ can be is ascribed to the variation in the configuration entropy of the EC and DEC molecules. $N_{\text {active }}$ gives us a useful strategy to design and/or search conjugated polymers with high $\alpha$.

Acknowledgments This work was supported by JSPS KAKENHI (Grant Nos. JP17H01137 and JP20J21687), the Murata Science Foundation and joint research with the Focus Systems Corporation.

1) S. W. Lee, Y. Yang, H.-W. Lee, H. Ghasemi, D. Kraemer, G. Chen, and Y. Cui, Nat. Commun. 5, 3942 (2014).

2) Y. Yang, S. W. Lee, H. Ghasemi, J. Loomis, X. Li, D. Kraemer, G. Zheng, Y. Cui, and G. Chen, PNAS 111, 17011 (2014).
3) J. Wang, S.-P. Feng, Y. Yang, N. Y. Hau, M. Munro, E. Ferreira-Yang, and G. Chen, Nano Lett. 15, 5784 (2015).

4) T. Shibata, Y. Fukuzumi, W. Kobayashi, and Y. Moritomo, Appl. Phys. Express 11, 017101 (2018).

5) Y. Fukuzumi, K. Amaha, W. Kobayashi, H. Niwa, and Y. Moritomo, Energy Technol. 6, 1 (2018).

6) T. Shibata, Y. Fukuzumi, and Y. Moritomo, Sci. Rep. 8, 14784 (2018).

7) I. Takahara, T. Shibata, Y. Fukuzumi, and Y. Moritomo, Chem. Select. 4, 8558 (2019).

8) T. Shibata, H. Iwaizumi, Y. Fukuzumi, and Y. Moritomo, Sci. Rep. 10, 1813 (2020).

9) Y. Fukuzumi, Y. Hinuma, and Y. Moritomo, Jpn. J. Appl. Phys. 58, 065501 (2019).

10) P. Novák, K. Müller, K. S. V. Santhanam, and O. Haas, Chem. Rev. 97, 207 (1997).

11) S. Muench, A. Wild, C. Friebe, B. Häupler, T. Janoschka, and U. S. Schubert, Chem. Rev. 116, 9438 (2016).

12) L. Wang, C. Pan, A. Liang, X. Zhou, W. Zhou, T. Wan, and L. Wang, Polym. Chem. 8, 4644 (2017).

13) J. Xie, P. Gu, and Q. Zhang, ACS Energy Lett. 2, 1985 (2017)

14) L. W. Shocklette, J. E. Toth, N. S. Murthy, and R. H. Baughman, J. Electrochem. Soc. 132, 1529 (1985).

15) M. Satoh, M. Tabata, K. Kateto, and K. Yoshino, Jpn. J. Appl. Phys. 25, L73 (1986).

16) K. Kateto, K. Yoshino, and Y. Inuishi, Jpn. J. Appl. Phys. 22, L567 (1983).

17) T. P. Kaloni, P. K. Giesbrecht, G. Schreckenbach, and M. S. Freund, Chem. Mater. 29, 10258 (2017).

18) H. Iwaizumi, T. Sugano, T. Yasuda, Y. Shimoi, W. Kobayashi, and Y. Moritomo, Jpn. J. Appl. Phys. 58, 097004 (2019).

19) T. Yasuda, T. Suzuki, M. Takahashi, and T. Tsutsui, Chem. Lett. 38, 1040 (2009).

20) Y. Furukawa, in Vibrational Spectroscopy of Conducting Polymers: Fundamentals and Applications in Vibrational Spectroscopy of Polymers: Principles and Practice, ed. N. J. Everall et al. (Wiley, New York, 2007).

21) N. Yokonuma, Y. Furukawa, M. Tasumi, M. Kuroda, and J. Nakayama, Chem. Phys. Lett. 255, 431 (1996)

22) K. Honda, Y. Furukawa, K. Furuya, H. Torii, and M. Tasumi, J. Phys. Chem. A 106, 3587 (2002).

23) A. Sakamoto, Y. Furukawa, and M. Tasumi, J. Phys. Chem. 98, 4635 (1994).

24) M. J. Frisch et al., Gaussian 16, Revision A.03 (Gaussian, Inc., Wallingford, CT, 2016).

25) D. Inoue, Y. Fukuzumi, and Y. Moritomo, Jpn. J. Appl. Phys. 59, 037001 (2020).

26) H. Iwaizumi, Y. Fujiwara, Y. Fukuzumi, and Y. Moritomo, Dalton Trans. 48, 1964 (2019).

27) D. E. Richardson and P. Sharpe, Inorg. Chem. 32, 1809 (1993)

28) G. Prampolini, P. Yu, S. Pizzanelli, I. Cacelli, F. Yang, J. Zhao, and J. Wang, J. Phys. Chem. 118, 14899 (2014) 